() А.Н. Кучер*, Н.П. Бабушкина

НИИ медицинской генетики, Томский национальный медицинский исследовательский центр, Томск

Метформин является одним из наиболее часто назначаемых для лечения и профилактики сахарного диабета 2 типа (СД2) лекарственных препаратов. Накапливаются многочисленные свидетельства, указывающие, что в основе неблагоприятных побочных эффектов метформина, а также индивидуальных различий ответа пациентов на лечение лежат как генетические, так и средовые факторы. В настоящем обзоре обобщена информация о генетических факторах и средовых модификаторах, определяющих индивидуальную реакцию пациентов на лечение метформином. Обобщены данные о роли полиморфизма наиболее значимых генов и кодируемых ими белковых продуктов как в развитии неблагоприятных эффектов, так и в определении терапевтического ответа, а также обсуждено место метформина в реализации фенотипических эффектов данных генов.

Рассмотрены эндогенные условия и экзогенные воздействия, модифицирующие ответ на метформин. Среди них факторы, влияющие на функциональное состояние генома (уровень метилирования генов, так или иначе связанных с ответом на метформин, и др.), отражающие биологические особенности организма (половая принадлежность, возраст), уровень здоровья (наличие сопутствующих заболеваний, обеспеченность организма биометаллами и витаминами), прием других лекарственных препаратов и т.д. Таким образом, существует широкий спектр модифицирующих ответ на метформин факторов, которые, в отличие от генетических особенностей, являются в большинстве своем управляемыми. При этом и генетические, и средовые факторы могут различаться по значимости в разных этно-территориальных группах населения. Данную информацию необходимо принимать во внимание при разработке персонализированного подхода к назначению метформина для лечения СД2, а также при рекомендации его использовать для лечения других болезней.

КЛЮЧЕВЫЕ СЛОВА: метформин; индивидуальный ответ; генетический полиморфизм; средовые факторы

\title{
ROLE OF GENETIC AND ENVIRONMENTAL FACTORS IN DETERMINING THE RESPONSE TO METFORMIN
}

\author{
(c) Aksana N. Kucher*, Nadezhda P. Babushkina
}

Research Institute of Medical Genetics, Tomsk National Research Medical Center, Tomsk, Russia

Metformin is one of the most commonly prescribed drugs for the treatment and prevention of type 2 diabetes mellitus (T2DM). Numerous evidence accumulates indicating that both genetic and environmental factors underlie adverse side effects of metformin, as well as individual differences in patient response to treatment The present review summarizes information on genetic factors and environmental modifiers determining patients' individual response to metformin treatment. The data on the role of polymorphism of the most significant genes and protein products encoded by them both in the development of adverse effects and in determining the therapeutic response are summarized, and the place of metformin in the realization of the phenotypic effects of these genes is discussed.

Endogenous conditions and exogenous effects modifying the response to metformin are considered. Among them are factors that affect the functional state of the genome (the level of methylation of genes, one way or another associated with the response to metformin, etc.), reflecting the biological characteristics of the organism (gender, age), health level (the presence of concomitant diseases, the supply of biometals and vitamins), taking other medications, etc. Thus, there is a wide range of factors modifying the response to metformin, which, unlike genetic characteristics, are largely controllable. At the same time, both genetic and environmental factors may differ in significance in different ethno-territorial groups of the population. This information should be taken into account when developing a personalized approach to prescribing metformin for the treatment of type 2 diabetes, as well as when recommending its use for the treatment of other diseases.

KEYWORDS: metformin; individual response; genetic polymorphism; environmental factors 
Метформин - препарат первой линии, назначаемый для лечения и профилактики сахарного диабета 2 типа (СД2), относится к группе бигуанидов. В качестве гипогликемизирующего средства метформин используют с начала 50-х гг. XX в., и в настоящее время он является одним из наиболее часто назначаемых лекарственных препаратов. В мире данный бигуанид применяют около 100 млн человек [1]. В Российской Федерации большинство больных СД2 используют метформин, доля таких пациентов возросла с 49,4\% в 2014 г. до 66,3\% в 2018 г. [2]. Несмотря на разработку новых гипогликемизирующих препаратов, благодаря длительному положительному опыту применения и невысокой стоимости метформин рекомендуют в качестве «базовой терапии» для всех пациентов с СД2 (при хорошей переносимости и отсутствии противопоказаний) [3-5]. В то же время у некоторых пациентов (у 20-30\%) при приеме данного бигуанида регистрируют побочные эффекты (прежде всего - нарушения со стороны работы желудочно-кишечного тракта, иногда развивается метформин-ассоциированный лактатацидоз), что может явиться причиной отказа от терапии метформином [6-16]. Кроме того, наблюдается различная эффективность лечения метформином СД2: в некоторых популяциях до 20-40\% пациентов не отвечают на прием метформина (не удается достигнуть целевого уровня гликированного гемоглобина - $\left.\mathrm{HbA}_{1 c}\right)[17,18]$.

В основе индивидуальных различий ответа на метформин или чувствительности к данному препарату могут лежать генетические особенности пациентов. K. Zhou и соавт. [19] установили, что коэффициент наследуемости для такого показателя, как снижение уровня $\mathrm{HbA}_{1 c}$ в ответ на прием метформина, составляет 34\%, а для показателя «способность достигать целевого уровня $\mathrm{HbA}_{1 c}$ » - 32\%, при этом генетический компонент ответа на метформин формируется за счет множества вариантов, локализованных по всему геному, каждый из которых оказывает небольшой или умеренный эффект. Персонализированный ответ на прием данного бигуанида подтвержден транскриптомными исследованиями, согласно которым, у здоровых доноров в лимфоцитах крови после приема метформина регистрируются индивидуально-специфичные изменения в экспрессии генов (в том числе и генов, продукты которых значимы для синтеза инсулина) [10]. В то же время, как следует из оценки коэффициента наследуемости [19], более чем на 65\% ответ на этот лекарственный препарат зависит от других (негенетических) факторов.

С учетом того, что метформин не только активно применяется для лечения СД2, но и накапливаются сведения о его плейотропных свойствах (в том числе о широком терапевтическом потенциале [20-25], значимости для увеличения продолжительности жизни [26], антитоксических эффектах [27]), важным представляется выявление причин, определяющих индивидуальные особенности ответа на прием данного лекарственного препарата. Это позволит использовать персонализированный подход при назначении метформина с учетом как генетических особенностей индивидов, так и других факторов, модифицирующих ответ на данный препарат.

Цель настоящего обзора заключается в обобщении информации о генетических и средовых факторах, определяющих ответ на метформин у пациентов, страдающих СД2.
В качестве источника информации использованы базы данных научных публикаций (PubMed, eLIBRARY. $\mathrm{RU})$, геномные, протеомные и другие базы (GWAS Catalog [28], UniProt [29], Ensembl [30], GTExPortal [31], OMIM [32], DRUGBANK [33], PharmGK [34]). Ключевым для поиска являлось слово «метформин», которое использовалось как самостоятельно, так и в сочетании со словами «гены», «генетический контроль», «побочные эффекты» и т.д. Гены и полиморфные варианты, ассоциированные с ответом на метформин, использовались в качестве ключевых слов в случае поиска информации об их функции и сфере компетенции. Предпочтение отдавалось исследованиям, выполненным в 2015-2020 гг.

\section{ГЕНЕТИЧЕСКИЕ ФАКТОРЫ, ОПРЕДЕЛЯЮЩИЕ ОТВЕТ НА МЕТФОРМИН}

Индивидуальный ответ на прием лекарственных препаратов (в том числе и метформина) может быть обусловлен структурно-функциональными особенностями генов, кодирующих транспортеры и рецепторы/мишени лекарств, а также метаболизирующие их ферменты и/или белки, обеспечивающие вывод лекарств из организма. Для выявления генетических факторов, определяющих ответ на метформин, наиболее часто использовался кандидатный подход [8, 35-37]. Широкогеномные ассоциативные исследования (GWAS) проводились реже, но они существенно расширили спектр генов, потенциально значимых для формирования индивидуального ответа на прием метформина [28, 38, 39]. К настоящему времени установлено несколько десятков генов, полиморфизм которых ассоциирован с параметрами, отражающими фармакокинетику и фармакодинамику метформина, эффективность его использования для лечения СД2 (табл. 1): зарегистрированы ассоциации с изменением в ответ на прием данного препарата уровня глюкозы натощак и после нагрузочного текста, с уровнями $\mathrm{HbA}_{1 c^{\prime}}$ инсулина и других показателей.

Наиболее часто к исследованию с использованием кандидатного подхода привлекались полиморфные варианты генов, белковые продукты которых участвуют в транспорте метформина; данные белки относятся к суперсемейству переносчиков растворенных веществ (SLC). С ответом на метформин зарегистрированы ассоциации ряда полиморфных вариантов в генах $S L C 22 A 1$, SLC22A2, SLC22A3, SLC2A2, SLC29A4, SLC47A1, SLC47A2 (см. табл. 1). Например, аллель C rs8192675 (интронный вариант гена SLC2A2) ассоциирован с индуцированным метформином снижением уровня $\mathrm{HbA}_{1 с}$ у европеоидов [1]. Авторы данного исследования заключили, что индивидов с гомозиготным генотипом по аллелю C rs8192675 можно лечить более низкими дозами препарата (вследствие чего будет регистрироваться меньше побочных эффектов), тогда как для лиц с генотипами ТТ допустимо назначение доз, превышающих обычно рекомендуемые для достижения целевых показателей углеводного обмена. В ответ на прием метформина среди китайских пациентов у обладателей аллеля A rs2289669 гена SLC47A1 выявили значимое снижение уровня $\mathrm{HbA}_{1 с}$ [40], а у лиц с генотипом AA rs622342 гена SLC22A1 зафиксировали более высокие уровни глюкозы натощак, $\mathrm{HbA}_{1 c}$ в плазме и показателя HOMA-IR, чем у носителей 
аллеля C [41]. У обладающих генотипом CC rs622342 ливанских пациентов, по сравнению с носителями других генотипов, в плазме регистрировали максимальную концентрацию метформина (которая сохранялась более продолжительное время после приема препарата); а через 3 мес после начала лечения для них было характерно более выраженное снижение уровня глюкозы натощак, через 6 мес - уровня $\mathrm{HbA}_{1 с}$. Эти результаты [41, 42] указывают на связь rs622342 гена SLC22A1 с фармакокинетикой метформина и вариабельностью терапевтической эффективности. Зарегистрированы ассоциации с ответом на метформин и других полиморфизмов (rs628031 и rs594709) гена SLC22A1 [37].

Поскольку в одних и тех же SLC-генах локализовано несколько вариантов, ассоциированных с ответом на метформин, ожидаемым является выявление неблагоприятных гаплотипов $[37,43]$. В частности, более высокие уровни $\mathrm{HbA}_{1 с}$ регистрировали у лиц с гаплотипами CGA и CAG по rs622342, rs628031 и rs594709 гена SLC22A1 по сравнению с самым распространенным гаплотипом AGA [37]. Вместе с тем разные варианты одного и того же гена могут показывать избирательное влияние на различные показатели гликемического ответа на метформин [42, 44]. Так, через 3 мес после начала приема данного препарата у пациентов с СД2, обладающих генотипами ТT rs1867351 и GG rs4709400, регистрировали большее снижение уровня постпрандиальной глюкозы в плазме, с генотипами СТ rs2297374, GG rs4709400 или GG rs628031 - большее снижение уровня глюкозы в плазме натощак, с генотипами ТT rs1867351, AA rs628031 или СТ rs2297374 - большее снижение уровня $\mathrm{HbA}_{1 c^{\prime}}$ чем у лиц с другими генотипами соответствующих полиморфизмов гена SLC22A1 (OCT1) [44].

Роль белков, кодируемых SLC-генами, в транспорте метформина доказана многочисленными исследованиями [48, 55-57]. Например, установлено, что вклад переносчика органических катионов 1 (ОСТ1, продукт гена SLC22A1) и транспортера моноаминов (РМАT, кодируется геном SLC29A4) в поглощение метформина через апикальную мембрану составляет примерно 25 и 20\% соответственно [56].

Интересен тот факт, что метформин может регулировать уровень экспрессии генов, продукты которых участвуют в его транспорте. Так, в клетках печени средний уровень метилирования и уровень метилирования промоторов генов - переносчиков метформина (SLC22A1, SLC22A3 и SLC47A1) был ниже у пациентов с СД2, принимавших данное лекарственное средство. Уровень метилирования генов связан с уровнем их экспрессии (снижение уровня метилирования приводит к увеличению экспрессии), а также с уровнем глюкозы в сыворотке крови и индексом массы тела [7]. Зависимость эффекта метформина от уровня экспрессии генов-переносчиков наблюдали в клетках жировой ткани: снижение уровня MATE1 (кодируется геном SLC47A1) и PMAT (продукт гена SLC29A4) ослабляло антилиполитический эффект метформина [58]. При этом для генов, кодирующих переносчики метформина, характерны тканеспецифичная экспрессия и в ряде случаев зависимость уровня экспрессии от генотипов [31], что может лежать в основе различных эффектов полиморфных вариантов в ответ на прием метформина в разных тканях и у лиц с разными генотипами, влияющими на уровень экспрессии.
Среди других генов, полиморфные варианты которых ассоциированы с ответом на метформин (см. табл. 1), более половины приходится на гены, кодирующие белки и ферменты, вовлеченные в поддержание гомеостаза глюкозы и/или секрецию инсулина (CAPN10, CSMD1, FMO5, GCKR, HNF1B, HNF4A, IRS1, KCNJ11, NEUROD1, PPARA, STK11, SP1, TCF7L2, PRKAA1 и PRKAA2) [29]. Например, с эффективностью приема пероральных противодиабетических препаратов (в основном комбинации метформина и сульфонилмочевины) ассоциирован rs1801278 в гене IRS1, приводящий к несинонимичной замене (G972R): для индивидов с гомозиготным генотипом R972R риск назначения инсулина в качестве дополнительной терапии был на $80 \%$ выше, чем для лиц с генотипом G972G [51]. Аллельные варианты некоторых генов, ассоциированных с ответом на метформин, предрасполагают к развитию инсулиннезависимого сахарного диабета (в том числе варианты в генах CAPN10, HNF1B, HNF4A и др.), а мутации - к развитию моногенных заболеваний, связанных с нарушением углеводного обмена (табл. 2).

В масштабном исследовании, выполненном в рамках программы профилактики диабета (Diabetes Prevention Program - DPP), показано, что аллельные варианты генов СД взрослого типа у молодых (MODY) могут влиять на ответ на инсулино-сенсибилизирующие вмешательства [50]. Авторы данного исследования установили, что в группе больных СД2 через год после назначения метформина и рекомендаций по изменению образа жизни была выявлена ассоциация минорного аллеля rs3212185 гена HNF4A с улучшением функции $\beta$-клеток, а минорного аллеля rs6719578 гена NEUROD1 - с увеличением секреции инсулина, но такой зависимости не наблюдали в группе плацебо [50]. После приема метформина в крови здоровых индивидов регистрировался более высокий уровень экспрессии HNF1A, HNF4A, NEUROD1, но для этих генов, а также гена KCNJ11 была характерна индивидуально-специфическая дифференциальная экспрессия, что, по мнению авторов исследования, хорошо согласуется с известной вариабельностью терапевтического ответа на принимаемый пациентами препарат [10]. Иными словами, ассоциации с ответом на метформин наблюдались также для полиморфных вариантов различных генов, которые потенциально могут выступать в качестве мишеней для метформина.

Принципиально важное значение для эффекта метформина имеют также гены PRKAA1 и PRKAA2, кодирующие две каталитические субъединицы АМФ-активированной протеинкиназы (АМФК), которая играет ключевую роль в обеспечении терапевтического эффекта метформина [59-62]. В частности, метформин подавляет продукцию глюкозы и экспрессию генов глюконеогенеза в первичных гепатоцитах через АМФК-зависимый механизм [61].

Несмотря на то что с ответом на метформин в основном установлены ассоциации с вариантами, локализованными в некодирующих регионах генов, большинство из них являются функционально значимыми, так как оказывают влияние на уровень экспрессии генов (eQTL) и/или на структуру кодируемого белкового продукта, изменяя сайты сплайсинга (sQTL) (см. табл. 1). Например, генотипы rs2076828, локализованного в 3'UTR гена SLC22A3, 


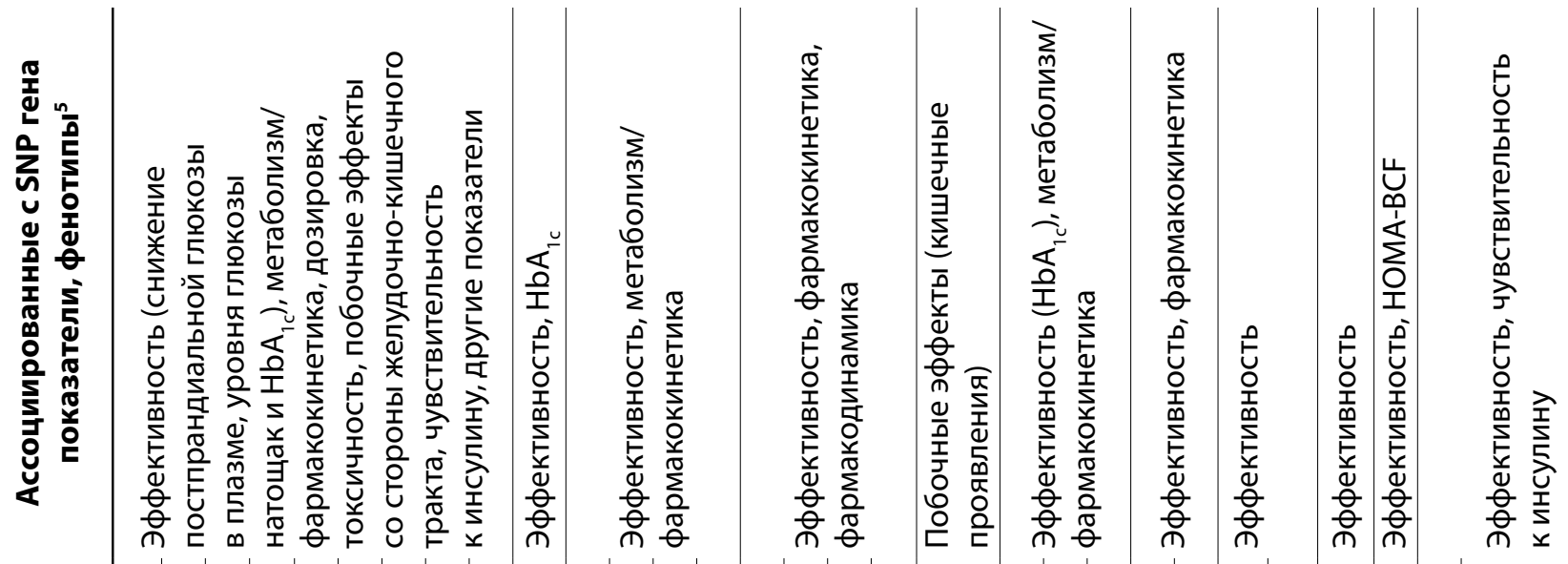
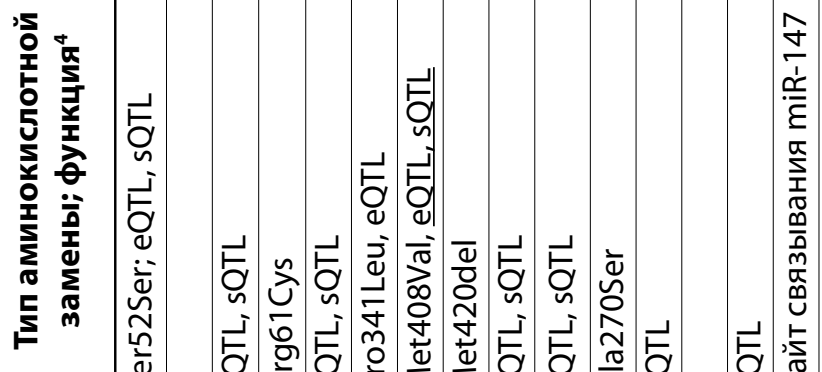

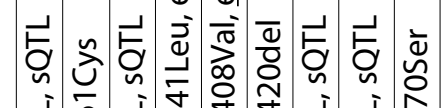

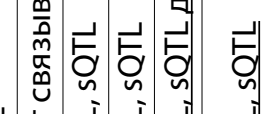

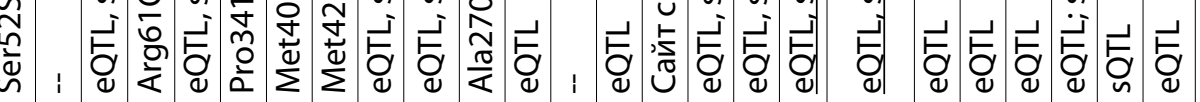

항ㅁㅇ

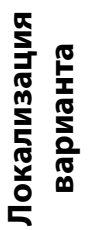

\section{乎 象}

$\stackrel{\Upsilon 2}{5}$

$\stackrel{0}{5}$

离

온

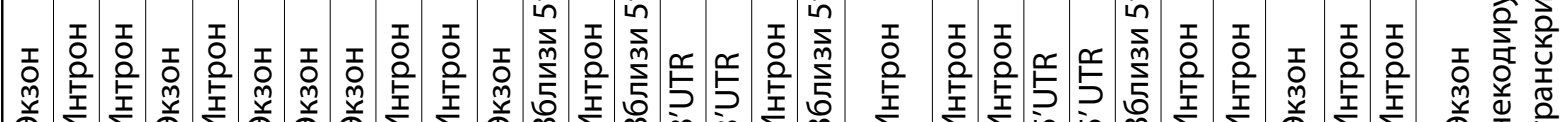

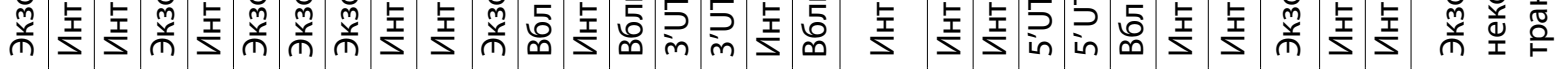

응 뭉

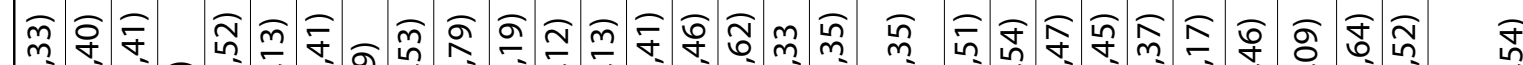

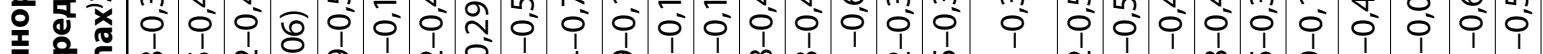

ร ㄴ.

萑

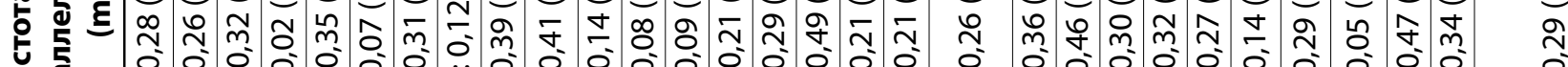

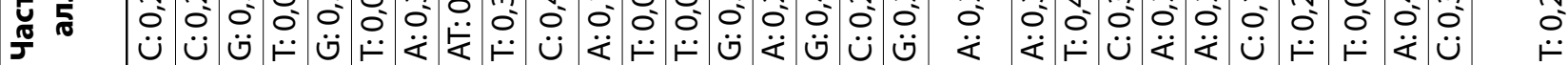

喜

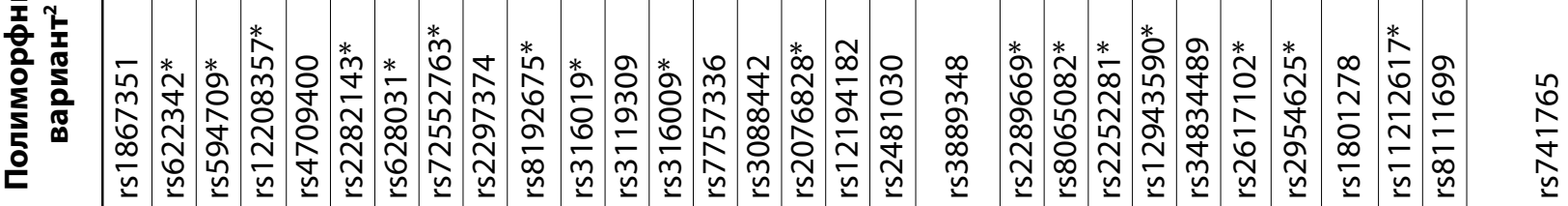

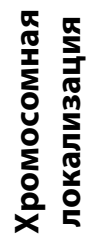

$\stackrel{m}{\stackrel{m}{n}} \underset{\delta}{\sigma}$

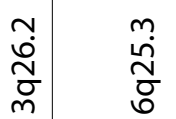

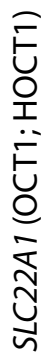

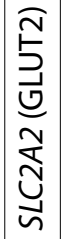

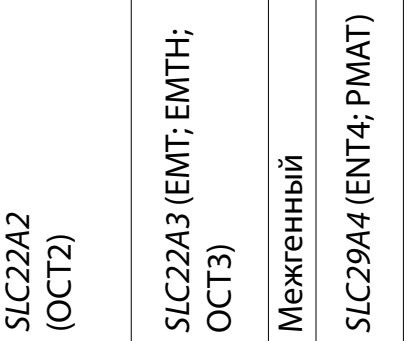

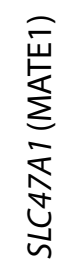

ปิ

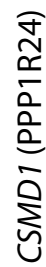

武痛 


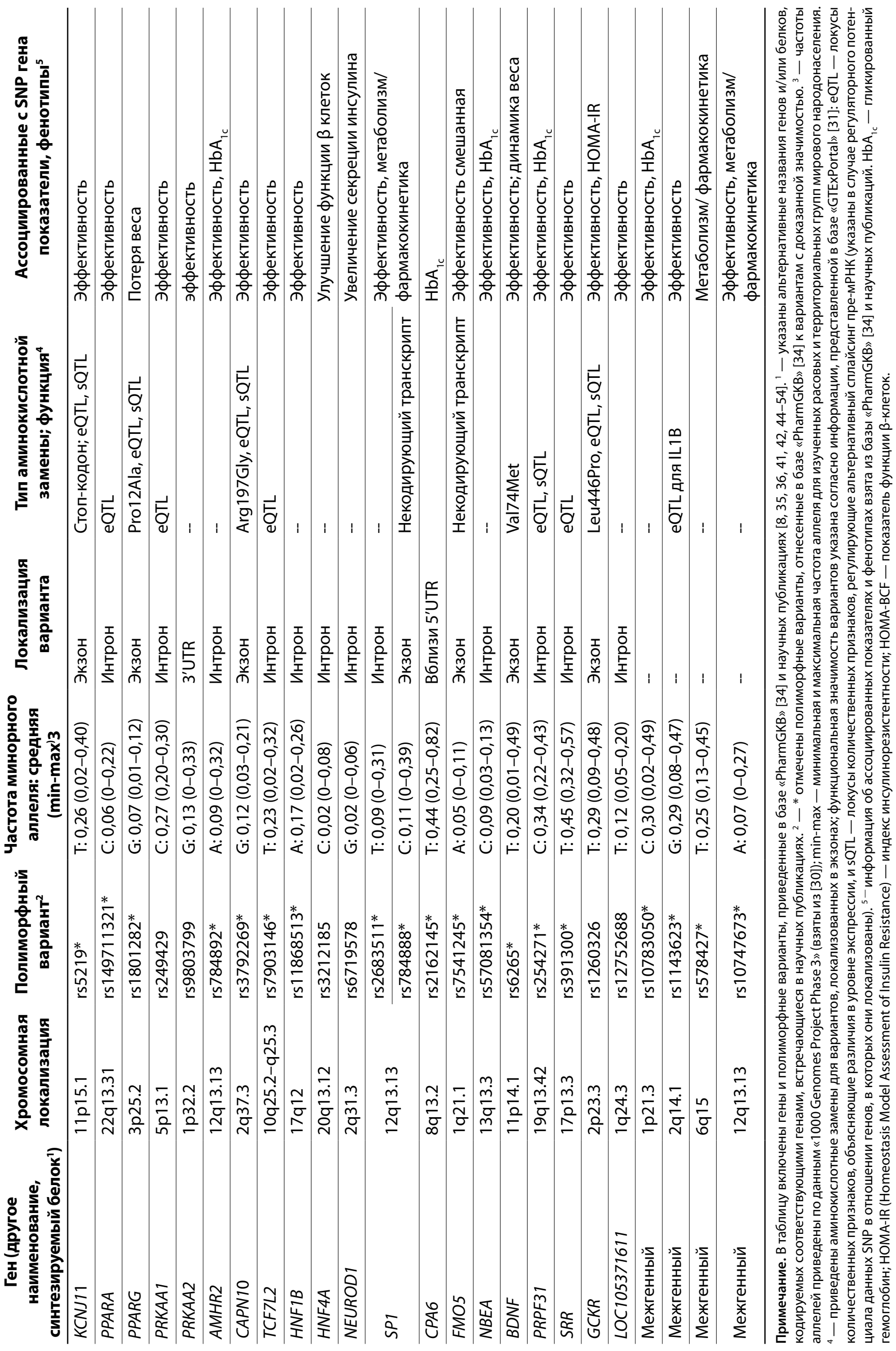


Таблица 2. Гены, ассоциированные с метформином, и их связь с заболеваниями/признаками, связанными с нарушением углеводного обмена (по [32])

\begin{tabular}{|c|c|c|c|}
\hline Ген & Патология & $\begin{array}{c}\text { Тип } \\
\text { наследования }\end{array}$ & MIM \\
\hline GCKP & Уровень глюкозы в плазме натощак (QTL5) & - & 613463 \\
\hline CAPN10 & Сахарный диабет инсулиннезависимый 1 & $\Pi P$ & 601283 \\
\hline \multirow{2}{*}{ PPARG } & Сахарный диабет, тип 2 & ПР-АД & 125853 \\
\hline & Тяжелая инсулинорезистентность, дигенная & АД & 604367 \\
\hline IRS1 & Сахарный диабет, тип 2 & ПР-АД & 125853 \\
\hline TCF7L2 & Сахарный диабет, тип 2 & ПР-АД & 125853 \\
\hline$S L C 2 A 2$ & Сахарный диабет инсулиннезависимый & АД & 125853 \\
\hline \multirow[b]{2}{*}{ HNF1B } & Сахарный диабет инсулиннезависимый & АД & 125853 \\
\hline & $\begin{array}{l}\text { Кисты почек и диабетический синдром / сахарный диабет взрослого } \\
\text { типа у молодых (MODY5) }\end{array}$ & АД & 137920 \\
\hline \multirow{3}{*}{ HNF4A } & Сахарный диабет инсулиннезависимый & ПР-АД & 125853 \\
\hline & MODY1 & АД & 125850 \\
\hline & $\begin{array}{l}\text { Ренотубулярный синдром Фанкони, тип 4, с диабетом зрелого } \\
\text { возраста у молодых }\end{array}$ & АД & 616026 \\
\hline \multirow{5}{*}{ KCNJ11 } & Сахарный диабет 2 типа & ПР-АД & 125853 \\
\hline & Семейная гиперинсулинемическая гипогликемия, тип 2 & $\mathrm{AP}$ & 601820 \\
\hline & $\begin{array}{l}\text { Неонатальный сахарный диабет, тип 2, с или без неврологических } \\
\text { особенностей }\end{array}$ & АД & 618856 \\
\hline & Преходящий неонатальный сахарный диабет, тип 3 & АД & 610582 \\
\hline & MODY13 & АД & 616329 \\
\hline \multirow{2}{*}{ NEUROD1 } & Сахарный диабет 2 типа & ПР-АД & 125853 \\
\hline & MODY6 & - & 606394 \\
\hline
\end{tabular}

Примечание. QTL — Quantitative Trait Locus — локус количественного признака; MODY — Maturity-Onset Diabetes of the Young — caхарный диабет взрослого типа у молодых; АД и АР — аутосомно-доминантный и аутосомно-рецессивный типы наследования соответственно; ПР — предрасположенность; ПР-АД — предрасположенность, реализующаяся по аутосомно-доминантному типу.

влияют на уровень мРНК, на активность кодируемого данным геном белка и, соответственно, на фармакодинамику метформина у здоровых добровольцев (ответ был хуже у лиц с минорным аллелем) [48]. Способность к поглощению и транспортировке метформина может зависеть от структурных особенностей белковых молекул, вовлеченных в этот процесс, как это было показано в случае несинонимичной замены R61C (rs12208357) и делеции M420del (rs72552763) в гене SLC22A1 [63]. В гене CAPN10 при rs3792269 происходит не только изменение в структуре кодируемого им белка (Arg197Gly), но этот же вариант обладает регуляторным потенциалом (eQTL, sQTL) [30, 31].

Ассоциированные с ответом на метформин генетические варианты различаются по уровню полиморфизма и по частоте регистрации аллелей в разных расово-территориальных группах населения, что предполагает наличие межэтнических различий в ответе на прием метформина (с точки зрения как эффективности лечения, так и развития побочных эффектов). Значительные различия по частоте регистрации минорного аллеля установлены для rs72552763 и rs72552763 гена SLC22A1, rs7757336 гена SLC22A2, rs2289669 гена SLC47A1, rs5219 гена KCNJ11, rs1260326 гена GCKR и др. (см. табл. 1). Однако указанные в табл. 1 полиморфные варианты как SLC-генов (всего приведена информация для 24 SNP), так и других генов, определяющих ответ на метформин, не отражают весь спектр вариантов, которые могут влиять на фармакокинетику, фармакодинамику метформина и, соответственно, на терапевтический ответ при приеме данного препарата. Только в гене SLC22A1 (OCT1) в 10 этнических группах в общей сложности описаны 34 полиморфных варианта, определяющих реакцию на метформин (наличие желудочно-кишечных побочных эффектов, уровень $\mathrm{HbA}_{1 c^{\prime}}$ уровень глюкозы в плазме натощак и после приема пищи), причем регистрировались межэтнические различия по частоте встречаемости аллелей данных полиморфизмов [64]. Кроме того, известны и другие переносчики метформина (например, SLC19A3, SLC2A3, SLC22A4, SLC6A4 [57]), и варианты в кодирующих их генах также могут оказывать влияние на индивидуальный ответ на данный лекарственный препарат.

Наряду с распространенными, редкие варианты, зачастую регистрируемые только в одной популяции, могут определять фармакодинамику и фармакокинетику метформина и вносить вклад в формирование ответа на прием данного препарата (например, rs34447885, rs34130495, локализованные в гене SLC22A1, rs145450955, rs201919874, локализованные в гене SLC22A2, rs77630697, rs7747426, rs35646404, локализованные в гене SLC47A1, rs373244724, rs562968062, rs146901447, локализованные в гене SLC47A2, и многие другие (см. [30, 52]). 
При секвенировании SLC-генов (изучены образцы более 140 тыс. индивидов из 7 основных групп населения мира) установлено более 204 тыс. однонуклеотидных вариантов, 99,8\% из них регистрировались с частотой менее 1\%, при этом каждый отдельный геном содержал в среднем 29,7 варианта с предполагаемым функциональным эффектом, из которых на долю редких приходилось 18\% [65]. Авторы данного исследования подчеркивают существенную межэтническую изменчивость по распространенности различных аллелей SLC-генов, причем 83\% патологических вариантов SLC-генов были выявлены только в одной популяции. С другой стороны, множественные полиморфные варианты с функциональными последствиями, включая M420del/ rs35167514 (SLC22A1*2), G401S/rs34130495 (SLC22A1*4) и G465R/rs34059508 (SLC22A1*5), а также R61C/rs12208357 $(S L C 22 A 1$ *3), не регистрируются у жителей Восточной Азии, но достигают существенной частоты (до 21,9\%) в иных популяциях [65]. При этом известно, что редкие варианты, по сравнению с часто регистрируемыми, могут оказывать более значимый эффект на формирование признаков/патологических фенотипов [66], что также подтверждает возможность этно-территориальных различий в ответе на метформин.

Спектр генов, белковые продукты которых влияют на чувствительность к метформину, гораздо шире перечня, представленного в табл. 1. Об этом может свидетельствовать тот факт, что метформин оказывает влияние на уровень метилирования более 100 генов, причем основные функции большинства из них связаны с хорошо известными мишенями для терапии метформином как в случае нарушения углеводного обмена, так и при других физиологических и патофизиологических процессах (в том числе регуляция энергетического гомеостаза, воспалительные реакции, онкогенез и нейродегенеративные процессы) [67]. Влияние метформина на экспрессию генов подтверждается данными транскриптомного анализа [10, 68-70]. M.R. Luizon и соавт. [69] идентифицировали тысячи чувствительных к метформину AMPK-зависимых и АMPK-независимых дифференциально экспрессирующихся генов и регуляторных элементов, что, по мнению авторов, может быть связано с межиндивидуальной вариабельностью гликемического ответа на метформин. Своеобразие генетических ландшафтов как по SLC-генам, так и по генам, чувствительным к воздействию метформина, как и вариабельность транскриптомных профилей в ответ на прием данного бигуанида, могут определять специфичность ответа на метформин у разных индивидов и в разных в этно-территориальных группах населения, что важно принимать во внимание при формировании региональных и персонифицированных терапевтических стратегий использования данного препарата.

Следует подчеркнуть, что выявленные ассоциации генетических маркеров с ответом на метформин являются неустойчивыми и воспроизводятся не во всех исследованиях (см., например, [34]). Более того, есть публикации, в которых указывается, что метформин может увеличивать риск развития СД2 [71], а для других заболеваний регистрируются разнонаправленные эффекты (положительный и отрицательный) в ответ на прием данного препарата. Такая ситуация, в частности, описа- на в отношении заболеваний сердечно-сосудистой системы [12, 72-75], в случае ответа на лечение ожирения у детей и подростков [24, 76], в определении характера течения инфекционного заболевания COVID-19 [77-79] и для других болезней. В качестве причины противоречивости могут выступать генетические особенности обследованных выборок пациентов. Так, N. Schweighofer и соавт. [73] отмечали, что повышенный риск общей смертности и смертности от сердечно-сосудистых заболеваний регистрируется у лиц с некоторыми генотипами по rs461473, rs609468, rs622591 гена SLC22A1 (OCT1), а как отмечалось выше, вариабельность по частоте регистрации аллелей может отличаться между разными этно-территориальными группами (см. [30]). Однако, с учетом оценок коэффициента наследуемости показателей, отражающих ответ на метформин (снижение $\mathrm{HbA}_{1 с}$ и способность достигать целевого уровня $\mathrm{HbA}_{1 c}$ ) [19], очевидна роль не только генетических, но и средовых факторов (в широком смысле этого слова) в детерминации терапевтического ответа на данный препарат, что также может объяснять противоречивость результатов его применения для лечения как СД2, так и других заболеваний.

\section{СРЕДОВЫЕ ФАКТОРЫ, МОДИФИЦИРУЮЩИЕ ОТВЕТ НА МЕТФОРМИН}

Ответ на метформин может зависеть от эндогенных условий и экзогенных воздействий. К числу модифицирующих ответ на метформин можно отнести факторы, которые влияют на функциональное состояние генома (например, уровень метилирования генов, так или иначе связанных с ответом на метформин), отражают биологические особенности организма (половая принадлежность, возраст), уровень здоровья (наличие сопутствующих заболеваний), прием других лекарственных препаратов и т.д.

Установлено, что, с одной стороны, метформин оказывает влияние на уровень метилирования ряда генов, с другой - статус метилирования генов информативен для выделения групп, по-разному реагирующих на прием данного препарата $[67,80]$. Так, зарегистрированы различия по уровню метилирования некоторых CpG-сайтов в клетках крови индивидов как между отвечающими и не отвечающими на метформин, так и между толерантными и чувствительными (неблагоприятные проявления) к данному лекарственному препарату (11 и 4 CpG сайта соответственно) [80]. Выявленные эпигенетические маркеры обладали высокой дифференцирующей способностью и отражали эпигенетический паттерн в жировой ткани (рассматривается в качестве ключевой ткани в патогенезе СД2), в то время как продукты генов, для которых были аннотированы данные эпигенетические маркеры, в гепатоцитах участвуют в биологических процессах, чувствительных к воздействию метформина.

Как уже отмечалось, метформин оказывает влияние на уровень метилирования SLC-генов, кодирующих белки-переносчики метформина [7]. Интересно, что у пациентов с диабетом, получавших метформин, метилирование ДНК генов SLC22A1, SLC22A3 и SLC47A1 было близко к таковому у лиц, не страдающих диабетом. При изначально высоком уровне метилирования SLC-генов (регистрируется у лиц с высоким уровнем сахара [7]), может 
нарушаться всасывание данного бигуанида в кишечнике, что, в свою очередь, способствует неблагоприятным кишечным проявлениям. В то же время после приема метформина происходят снижение уровня метилирования и увеличение экспрессии SLC-генов, продукты которых участвуют в транспорте данного препарата [7]. Это согласуется с клиническими наблюдениями, согласно которым при приеме данного препарата в ряде случаев неблагоприятные кишечные проявления у пациентов со временем уменьшаются.

На риск развития неблагоприятных эффектов метформина могут влиять сопутствующие заболевания $[6,11,13]$. Y. Huang и соавт. [6] установили, что к побочным эффектам метформина может приводить бессимптомный (или недифференцированный) гастрит. У лиц, страдающих данной патологией, при приеме метформина была выше частота неблагоприятных желудочно-кишечных проявлений и меньше средняя конечная доза метформина по сравнению с не страдающими гастритом (последний показатель оказался не значим после введения поправки на пол и возраст, которые также можно рассматривать как модифицирующие факторы). Риск развития лактатацидоза и вероятность смертельного исхода при данном состоянии возрастают у больных СД2, имеющих сопутствующие заболевания (сепсис, почечная недостаточность и др.) $[11,13]$. В случае нарушения работы почек (через которые выводится метформин из организма) риск побочных эффектов увеличивается в связи с увеличением периода полувыведения этого бигуанида из крови и уменьшения почечного клиренса [33].

Прием пациентами с сопутствующими заболеваниями лекарственных препаратов, ингибирующих белки-переносчики метформина или использующих их для собственной фармакодинамики, является еще одним фактором риска развития неблагоприятных эффектов метформина, таких как кишечные симптомы, лактатацидоз, снижение уровня витамина $\mathrm{B}_{12}$ (на уровень данного витамина также могут оказывать влияние различные лекарственные препараты) [8, 13, 81, 82]. К числу таких препаратов относятся некоторые диуретики, ингибиторы АПФ; ингибиторы протонной помпы, селективные антагонисты гистаминовых рецепторов H2RA, нестероидные противовоспалительные средства и другие. Однако среди лекарственных препаратов есть как снижающие, так и увеличивающие концентрацию метформина в сыворотке крови [33]. В частности, при приеме одновременно с метформином ацетилсалициловой кислоты увеличивается риск развития или тяжесть гипогликемии, при приеме альдостерона, напротив, увеличивается риск и тяжесть гипергликемии, ацикловир снижает уровень выведения метформина и способствует увеличению его концентрации в сыворотке крови и т.д. Прием таких препаратов, влияющих на уровень метформина в крови, может объяснять определенный процент случаев как толерантности, так и непереносимости данного бигуанида у пациентов с СД2. Полиморбидность и, соответственно, полипрагмазия могут выступать в качестве одной из причин того, что у пожилых лиц чаще регистрируют неблагоприятные кишечные проявления при приеме метформина [8].

На эффективность применения метформина может оказывать влияние обеспеченность организма биометаллами и витаминами. Так, показано, что клеточные эффекты метформина зависят от его способности связывать ионы меди, а недостаток данного элемента приводит к нарушению передачи сигналов через АМР-активированную протеинкиназу [83]. Известно также, что метформин усиливает экспрессию гена СР, кодирующего церулоплазмин - гликопротеин, связывающий медь [84]; одна из функций данного белка состоит в поддержании клеточного гомеостаза железа [29]. Следует подчеркнуть, что многие гены, полиморфные варианты которых показали ассоциации с эффективностью и/или переносимостью метформина, кодируют белки, функционально связанные с ионами металлов или витаминами [29], в том числе и теми, дефицит которых значим для развития нарушений углеводного обмена $[85,86]$. Например, в качестве кофакторов ионы цинка выступают для CPA6, ионы меди и магния - для STK11, AMHR2, ионы магния - для PRKAA1 и PRKAA2, витамин $\mathrm{B}_{6}-$ для SRR; роль лигандов играют ионы цинка для PPARA, PPARG, HNF4A, SP1 [29].

С другой стороны, M. Wakeman и D.T. Archer [82] на основании обобщения научных публикаций заключили, что использование метформина может оказывать влияние на уровень витаминов группы В (в том числе $\mathrm{B}_{12}$ ), витамина $\mathrm{D}$ и магния, а также приводить к изменениям в составе микробиома. В этой связи интересным представляется исследование R. Krysiak и соавт. [87], в котором показано, что у индивидов с преддиабетом, страдающих тиреоидитом Хашимото, одновременный прием метформина и витамина D приводил к более значительному снижению уровня глюкозы в плазме и улучшению чувствительности к инсулину (а также к снижению уровня тиреотропина и титра антител к тиреопероксидазе), чем у лиц, принимавших только метформин.

Значимость дозы и длительность применения метформина для риска развития неблагоприятных эффектов обсуждается в ряде исследований $[15,88,82]$. Для эффективного применения препарата также важно учитывать назначение дозы метформина в зависимости от функционального состояния организма [89]. В частности, женщинам с инсулинорезистентностью и синдромом поликистозных яичников, у которых был высоким индекс массы тела, потребовалась большая доза метформина, чтобы достичь хорошего ответа на терапию (включая снижение индекса массы тела, нормализацию менструального цикла, снижение уровня холестерина и гиперандрогении) [89].

Установлено, что метформин оказывает влияние на микробиом кишечника [90-92]. На модельных объектах (на мышах) показано, что толерантность к глюкозе улучшалась у особей, получивших измененную метформином микробиоту [90]. Изменение микробиоты у принимавших метформин сопровождалось также изменением в метаболомике: в частности, регистрировался более высокий уровень бутирата и пропионата, участвующих в поддержании гомеостаза глюкозы [92]. Из этого следует, что на толерантность или непереносимость метформина человека может влиять его микробиом [90, 92]. Интересно, что в другом исследовании [93] наблюдали более высокие концентрации микробного метаболита имидазола пропионата у принимающих метформин пациентов, страдающих СД2, с высоким уровнем глюкозы в крови. 
И, конечно, стоит указать на такие хорошо известные модификаторы эффектов метформина, как прием препарата во время еды (снижает риск неблагоприятных кишечных проявлений) и употребление алкоголя (увеличивает риск лактатацидоза).

Приведенные данные указывают на широкий спектр модифицирующих ответ на метформин факторов, которые, в отличие от генетических особенностей, являются в большинстве своем управляемыми.

\section{ЗАКЛЮЧЕНИЕ}

Таким образом, ответ на прием метформина у пациентов, страдающих СД2, зависит от их генетических особенностей и эпигенетических модификаций генов, отвечающих за транспорт метформина или являющихся его мишенями, эндогенных (возраст, наличие сопутствующих заболеваний, обеспеченность некоторым витаминами и биометаллами, особенности кишечной микрофлоры) и экзогенных (доза и продолжительность приема метформина, полипрагмазия) средовых факто- ров. И генетические, и средовые факторы могут различаться по значимости в разных этно-территориальных группах населения. Это важно принимать во внимание при разработке персонализированного подхода к назначению метформина для лечения СД2, а также при рекомендации его использовать для лечения других болезней.

\section{ДОПОЛНИТЕЛЬНАЯ ИНФОРМАЦИЯ}

Источники финансирования. Работа выполнена при частичном финансировании Госзадания Министерства науки и высшего образования № 075-00603-19-00.

Конфликт интересов. Авторы декларируют отсутствие явных и потенциальных конфликтов интересов, связанных с содержанием настоящей статьи.

Участие авторов. Кучер А.Н. - поиск литературы, написание рукописи, редактирование и финальное утверждение рукописи; Бабушкина Н.П. - поиск литературы, редактирование текста, подготовка рукописи к публикации. Все авторы внесли существенный вклад в подготовку статьи, прочли и одобрили финальную версию перед публикацией.

\section{СПИСОК ЛИТЕРАТУРЫ | REFERENCES}

1. Zhou K, Yee SW, Seiser EL, et al. Variation in the glucose transporter gene SLC2A2 is associated with glycemic response to metformin. Nat Genet. 2016;48(9):1055-1059. doi: https://doi.org/10.1038/ng.3632

2. Дедов И.И., Шестакова М.В., Викулова О.К., и др. Атлас регистра сахарного диабета Российской Федерации. Статус 2018 г. // Сахарный диабет. - 2019. - T. 22. - № S2-2. - C. 4-61. [Dedov II, Shestakova MV, Vikulova OK, et al. Atlas of Diabetes Register in Russian Federation, status 2018. Diabetes Mellitus. 2019;22(2S):4-61. (In Russ.)]. doi: https://doi.org/10.14341/DM20192S

3. Аметов А.С., Демидова Т.Ю., Кочергина И.И. Эффективность препаратов метформина в лечении сахарного диабета 2-го типа // Медицинский совет. - 2016. - № 3. - С. 30-37. [Ametov AS, Demidova TY, Kochergina II. The efficacy of metformin in the treatment of type 2 diabetes. Med Counc. 2016;284(3):30-37. (In Russ)]. doi: https://doi.org/10.21518/2079-701X-2016-3-30-37

4. Inzucchi SE. Is It Time to Change the Type 2 Diabetes Treatment Paradigm? No! Metformin Should Remain the Foundation Therapy for Type 2 Diabetes. Diabetes Care. 2017;40(8):1128-1132. doi: https://doi.org/10.2337/dc16-2372

5. Дедов И.И., Шестакова М.В., Майоров А.Ю., и др. Сахарный диабет 2 типа у взрослых // Сахарный дuaбem. - 2020. - T. 23. — № S2. - C. 4-102. [Dedov II, Shestakova MV, Majorov AY, et al. Diabetes mellitus type 2 in adults. Diabetes Mellitus. 2020;23(S2):4-102. (In Russ.)] doi: https://doi.org/10.14341/DM20202S

6. Huang Y, Sun J, Wang $X$, et al. Asymptomatic chronic gastritis decreases metformin tolerance in patients with type 2 diabetes. J Clin Pharm Ther. 2015;40(4):461-465. doi: https://doi.org/10.1111/jcpt.12290

7. García-Calzón S, Perfilyev A, Männistö V, et al. Diabetes medication associates with DNA methylation of metformin transporter genes in the human liver. Clin Epigenetics. 2017;9:102. doi: https://doi.org/10.1186/s13148-017-0400-0

8. Dawed AY, Zhou K, van Leeuwen N, et al. Variation in the Plasma Membrane Monoamine Transporter (PMAT) (Encoded by SLC29A4) and Organic Cation Transporter 1 (OCT1) (Encoded by SLC22A1) and Gastrointestinal Intolerance to Metformin in Type 2 Diabetes: An IMI DIRECT Study. Diabetes Care. 2019;42(6):1027-1033. doi: https://doi.org/10.2337/dc18-2182

9. Мадянов И.В., Диарова А.А., Воронина М.Г., Долгова М.В. К вопросу о нежелательных реакциях при приеме метформина // Клиническая медицина и фармакология. 2019. - T. 5. — № 2. - C. 16-19. [Madjanov IV,
Diarova AA, Voronina MG, Dolgova MV. On the issue of adverse reactions when taking metformin. Klinicheskaja medicina i farmakologija. 2019;5(2):16-19. (In Russ.)]. doi: https://doi.org/10.12737/article_5d6620b2380101.83928732

10. Ustinova M, Silamikelis I, Kalnina I, et al. Metformin strongly affects transcriptome of peripheral blood cells in healthy individuals. PLoS One. 2019;14(11):e0224835. doi: https://doi.org/10.1371/journal.pone.0224835

11. Blumenberg A, Benabbas R, Sinert R, et al. Do Patients Die with or from Metformin-Associated Lactic Acidosis (MALA)? Systematic Review and Meta-analysis of $\mathrm{pH}$ and Lactate as Predictors of Mortality in MALA. J Med Toxicol. 2020;16(2):222-229. doi: https://doi.org/10.1007/s13181-019-00755-6

12. Hansen CS, Lundby-Christiansen L, Tarnow L, et al. Metformin may adversely affect orthostatic blood pressure recovery in patients with type 2 diabetes: substudy from the placebo-controlled Copenhagen Insulin and Metformin Therapy (CIMT) trial. Cardiovasc Diabetol. 2020;19(1):150. doi: https://doi.org/10.1186/s12933-020-01131-3

13. Schädle P, Tschritter O, Kellerer M. Metformin Associated Lactic Acidosis in Clinical Practice - A Case Series. Exp Clin Endocrinol Diabetes. 2020. doi: https://doi.org/10.1055/a-1149-9030.

14. Sendil S, Yarlagadda K, Lawal H, et al. Metformin Associated Lactic Acidosis in the Intensive Care Unit: A Rare Condition Mimicking Sepsis. Cureus. 2020;12(7):e9119. doi: https://doi.org/10.7759/cureus.9119

15. Shivaprasad C, Gautham K, Ramdas B, et al. Metformin Usage Index and assessment of vitamin B12 deficiency among metformin and non-metformin users with type 2 diabetes mellitus. Acta Diabetol. 2020;57(9):1073-1080. doi: https://doi.org/10.1007/s00592-020-01526-4

16. Theobald J, Schneider J, Cheema N, DesLauriers C. Time to development of metformin-associated lactic acidosis. Clin Toxicol (Phila). 2020;58(7):758-762. doi: https://doi.org/10.1080/15563650.2019.1686514

17. Cook MN, Girman CJ, Stein PP, Alexander CM. Initial monotherapy with either metformin or sulphonylureas often fails to achieve or maintain current glycaemic goals in patients with Type 2 diabetes in UK primary care. Diabet Med. 2007;24(4):350-8. doi: https://doi.org/10.1111/j.1464-5491.2007.02078.x

18. Rashid M, Shahzad M, Mahmood S, Khan K. Variability in the therapeutic response of Metformin treatment in patients with type 2 diabetes mellitus. Pak J Med Sci. 2019;35(1):71-76. doi: https://doi.org/10.12669/pjms.35.1.100. 
19. Zhou K, Donnelly L, Yang J, et al. Heritability of variation in glycaemic response to metformin: a genome-wide complex trait analysis. Lancet Diabetes Endocrinol. 2014;2(6):481-487. doi: https://doi.org/10.1016/S2213-8587(14)70050-6.

20. Бирюкова Е.В., Морозова И.А. Глюкофаж лонг — эффективный и безопасный сахароснижающий препарат для длительного применения // Эффективная фармакотерапия. - 2015. № 28. - C. 14-23. [Biryukova EV, Morozova IA. Glucophage Long - an Effective and Safe Sugar-Lowering Drug for a LongTerm Administration. Jeffektivnaja farmakoterapija. 2015;28:14-23. (In Russ.)].

21. Sung CT, Chao T, Lee A, et al. Oral Metformin for Treating Dermatological Diseases: A Systematic Review. J Drugs Dermatol. 2020;19(8):713-720. doi: https://doi.org/10.36849/JDD.2020.4874.

22. Dludla PV, Nkambule BB, Mazibuko-Mbeje SE, et al. Adipokines as a therapeutic target by metformin to improve metabolic function: A systematic review of randomized controlled trials. Pharmacol Res. 2021;163:105219. doi: https://doi.org/10.1016/j.phrs.2020.105219

23. Lv Z, Guo Y. Metformin and Its Benefits for Various Diseases. Front Endocrinol (Lausanne). 2020;11:191 doi: https://doi.org/10.3389/fendo.2020.00191

24. Sadeghi A, Mousavi SM, Mokhtari T, et al. Metformin Therapy Reduces Obesity Indices in Children and Adolescents: A Systematic Review and Meta-Analysis of Randomized Clinical Trials. Child Obes. 2020;16(3):174-191. doi: https://doi.org/10.1089/chi.2019.0040

25. Zhang QQ, Li WS, Liu Z, et al. Metformin therapy and cognitive dysfunction in patients with type 2 diabetes: A meta-analysis and systematic review. Medicine (Baltimore). 2020;99(10):e19378. doi: https://doi.org/10.1097/MD.0000000000019378.

26. Fang J, Yang J, Wu X, et al. Metformin alleviates human cellular aging by upregulating the endoplasmic reticulum glutathione peroxidase 7. Aging Cell. 2018;17(4):e12765. doi: https://doi.org/10.1111/acel.12765

27. Meshkani SE, Mahdian D, Abbaszadeh-Goudarzi K, et al. Metformin as a protective agent against natural or chemical toxicities: a comprehensive review on drug repositioning. J Endocrinol Invest. 2020;43(1):1-19. doi: https://doi.org/10.1007/s40618-019-01060-3

28. The NHGRI-EBI Catalog of human genome-wide association studies [Internet]. [cited 12.2020] Available from: https://www.ebi.ac.uk/gwas

29. UniProt [Internet]. Available from: https://www.uniprot.org [cited 10.2020]

30. Ensembl [Internet]. Available from: https://www.ensembl.org/ [cited 10.2020]

31. GTExPortal [Internet]. Available from: https://gtexportal.org/ [cited 10.2020]

32. OMIM - Online Mendelian Inheritance in Man [Internet] Available from: https://www.omim.org/ [cited 12.2020]

33. DRUGBANK. [Internet]. URL: https://go.drugbank.com/ [cited 12.2020]

34. PharmGKB [Internet]. Available from: https://www.pharmgkb.org/ chemical/PA450395/variantAnnotation [cited 10.2020]

35. Jablonski KA, McAteer JB, de Bakker PI, et al. Common variants in 40 genes assessed for diabetes incidence and response to metformin and lifestyle intervention in the diabetes prevention program. Diabetes. 2010;59(10):2672-2681. doi: https://doi.org/10.2337/db10-0543.

36. Al-Eitan LN, Almomani BA, Nassar AM, et al. Metformin Pharmacogenetics: Effects of SLC22A1, SLC22A2, and SLC22A3 Polymorphisms on Glycemic Control and HbA1c Levels. J Pers Med. 2019;9(1):17. doi: https://doi.org/10.3390/jpm9010017

37. Reséndiz-Abarca CA, Flores-Alfaro E, Suárez-Sánchez F, et al. Altered Glycemic Control Associated With Polymorphisms in the SLC22A1 (OCT1) Gene in a Mexican Population With Type 2 Diabetes Mellitus Treated With Metformin: A Cohort Study. J Clin Pharmacol. 2019;59(10):1384-1390. doi: https://doi.org/10.1002/jcph.1425

38. Chan P, Shao L, Tomlinson B, et al. Metformin transporter pharmacogenomics: insights into drug disposition-where are we now? Expert Opin Drug Metab Toxicol. 2018;14(11):1149-1159. doi: https://doi.org/10.1080/17425255.2018.1541981

39. Srinivasan S, Yee SW, Giacomini KM. Pharmacogenetics of Antidiabetic Drugs. Adv Pharmacol. 2018;83:361-389. doi: https://doi.org/10.1016/bs.apha.2018.04.005

40. Liang $\mathrm{H}, \mathrm{Xu}$ W, Zhou $\mathrm{L}$, et al. Differential increments of basal glucagon-like-1 peptide concentration among SLC47A1 rs2289669 genotypes were associated with inter-individual variability in glycaemic response to metformin in Chinese people with newly diagnosed Type 2 diabetes. Diabet Med. 2017;34(7):987-992. doi: https://doi.org/10.1111/dme.13351

41. Wu K, Li X, XU Y, et al. SLC22A1 rs622342 Polymorphism Predicts Insulin Resistance Improvement in Patients with Type 2 Diabetes Mellitus Treated with Metformin: A CrossSectional Study. Int J Endocrinol. 2020;2020:2975898. doi: https://doi.org/10.1155/2020/2975898

42. Naja K, El Shamieh S, Fakhoury R. rs622342A>C in SLC22A1 is associated with metformin pharmacokinetics and glycemic response. Drug Metab Pharmacokinet. 2020;35(1):160-164. doi: https://doi.org/10.1016/j.dmpk.2019.10.007

43. Marta M, Sánchez-Pozos K, Jaimes-Santoyo J, et al. Pharmacogenetic Evaluation of Metformin and Sulphonylurea Response in Mexican Mestizos with Type 2 Diabetes. Curr Drug Metab. 2020;21(4):291-300. doi: https://doi.org/10.2174/1389200221666200514125443

44. Zhou Y, Ye W, Wang Y, et al. Genetic variants of OCT1 influence glycemic response to metformin in Han Chinese patients with type-2 diabetes mellitus in Shanghai. Int J Clin Exp Pathol. 2015;8(8):9533-9542

45. Chen L, Takizawa M, Chen E, et al. Genetic polymorphisms in organic cation transporter 1 (OCT1) in Chinese and Japanese populations exhibit altered function. J Pharmacol Exp Ther. 2010;335(1):42-50. doi: https://doi.org/10.1124/jpet.110.170159

46. López-Bermejo A, Díaz M, Morán E, et al. A single nucleotide polymorphism in STK11 influences insulin sensitivity and metformin efficacy in hyperinsulinemic girls with androgen excess. Diabetes Care. 2010;33(7):1544-1548. doi: https://doi.org/10.2337/dc09-1750

47. Pollin TI, Jablonski KA, McAteer JB, et al. Diabetes Prevention Program Research Group. Triglyceride response to an intensive lifestyle intervention is enhanced in carriers of the GCKR Pro446Leu polymorphism. J Clin Endocrinol Metab. 2011;96(7):1142-1147. doi: https://doi.org/10.1210/jc.2010-2324

48. Chen EC, Liang $X$, Yee SW, et al. Targeted disruption of organic cation transporter 3 attenuates the pharmacologic response to metformin. Mol Pharmacol. 2015;88(1):75-83. doi: https://doi.org/10.1124/mol.114.096776.

49. Zaharenko L, Kalnina I, Geldnere K, et al. Single nucleotide polymorphisms in the intergenic region between metformin transporter OCT2 and OCT3 coding genes are associated with short-term response to metformin monotherapy in type 2 diabetes mellitus patients. Eur J Endocrinol. 2016;175(6):531-540. doi: https://doi.org/10.1530/EJE-16-0347

50. Billings LK, Jablonski KA, Warner AS, et al. Variation in Maturity-Onset Diabetes of the Young Genes Influence Response to Interventions for Diabetes Prevention. J Clin Endocrinol Metab. 2017;102(8):2678-2689. doi: https://doi.org/10.1210/jc.2016-3429

51. Prudente S, Di Paola R, Pezzilli S, et al. Pharmacogenetics of oral antidiabetes drugs: evidence for diverse signals at the IRS1 locus. Pharmacogenomics J. 2018;18(3):431-435. doi: https://doi.org/10.1038/tpj.2017.32

52. Mannino GC, Andreozzi F, Sesti G. Pharmacogenetics of type 2 diabetes mellitus, the route toward tailored medicine. Diabetes Metab Res Rev. 2019;35(3):3109. doi: https://doi.org/10.1002/dmrr.3109

53. Moeez S, Riaz S, Masood N, et al. Evaluation of the rs3088442 G>A SLC22A3 Gene Polymorphism and the Role of microRNA 147 in Groups of Adult Pakistani Populations With Type 2 Diabetes in Response to Metformin. Can J Diabetes. 2019;43(2):128-135. doi: https://doi.org/10.1016/j.jcjd.2018.07.001

54. Xhakaza L, Abrahams-October Z, Pearce B, et al. Evaluation of the suitability of 19 pharmacogenomics biomarkers for individualized metformin therapy for type 2 diabetes patients. Drug Metab Pers Ther. 2020. doi: https://doi.org/10.1515/dmdi-2020-0111

55. Zhou M, Xia L, Wang J. Metformin transport by a newly cloned proton-stimulated organic cation transporter (plasma membrane monoamine transporter) expressed in human intestine. Drug Metab Dispos. 2007;35(10):1956-1562. doi: https://doi.org/10.1124/dmd.107.015495

56. Han TK, Proctor WR, Costales CL, et al. Four cationselective transporters contribute to apical uptake and accumulation of metformin in Caco-2 cell monolayers. J Pharmacol Exp Ther. 2015;352(3):519-528. doi: https://doi.org/10.1124/jpet.114.220350 
57. Liang X, Giacomini KM. Transporters Involved in Metformin Pharmacokinetics and Treatment Response. J Pharm Sci. 2017;106(9):2245-2250. doi: https://doi.org/10.1016/j.xphs.2017.04.078

58. Arner P, Kulyte A, Batchelor K, et al. Mapping of biguanide transporters in human fat cells and their impact on lipolysis. Diabetes Obes Metab. 2018;20(10):2416-2425. doi: https://doi.org/10.1111/dom.13395

59. Zhou G, Myers R, Li Y, et al. Role of AMP-activated protein kinase in mechanism of metformin action. J Clin Invest. 2001;108(8):1167-1174. doi: https://doi.org/10.1172/JCl13505.

60. Hardie DG. AMPK: a target for drugs and natural products with effects on both diabetes and cancer. Diabetes. 2013;62(7):2164-2172 doi: https://doi.org/10.2337/db13-0368

61. Cao J, Meng S, Chang E, et al. Low concentrations of metformin suppress glucose production in hepatocytes through AMP-activated protein kinase (AMPK). J Biol Chem. 2014;289(30):20435-20446. doi: https://doi.org/10.1074/jbc.M114.567271

62. Meng S, Cao J, He Q, et al. Metformin activates AMPactivated protein kinase by promoting formation of the $a \beta y$ heterotrimeric complex. J Biol Chem. 2015;290(6):3793-3802. doi: https://doi.org/10.1074/jbc.M114.604421

63. Ahlin G, Chen L, Lazorova L, et al. Genotype-dependent effects of inhibitors of the organic cation transporter, OCT1: predictions of metformin interactions. Pharmacogenomics J. 2011;11(6):400-411. doi: https://doi.org/10.1038/tpj.2010.54

64. Mofo Mato EP, Guewo-Fokeng M, Essop MF, Owira PMO. Genetic polymorphisms of organic cation transporter 1 (OCT1) and responses to metformin therapy in individuals with type 2 diabetes: A systematic review. Medicine (Baltimore). 2018;97(27):e11349. doi: https://doi.org/10.1097/MD.0000000000011349

65. Schaller L, Lauschke VM. The genetic landscape of the human solute carrier (SLC) transporter superfamily. Hum Genet. 2019;138(1112):1359-1377. doi: https://doi.org/10.1007/s00439-019-02081-x

66. Bodmer W, Bonilla C. Common and rare variants in multifactorial susceptibility to common diseases. Nat Genet. 2008;40(6):695-701. doi: https://doi.org/10.1038/ng.f.136

67. Elbere I, Silamikelis I, Ustinova M, et al. Significantly altered periphera blood cell DNA methylation profile as a result of immediate effect of metformin use in healthy individuals. Clin Epigenetics. 2018;10(1):156. doi: https://doi.org/10.1186/s13148-018-0593-x

68. He J, Wang K, Zheng N, et al. Metformin suppressed the proliferation of LoVo cells and induced a time-dependent metabolic and transcriptional alteration. Sci Rep. 2015;5:17423. doi: https://doi.org/10.1038/srep17423

69. Luizon MR, Eckalbar WL, Wang Y, et al. Genomic Characterization of Metformin Hepatic Response. PLoS Genet. 2016;12(11):e1006449. doi: https://doi.org/10.1371/journal.pgen.1006449

70. Padilla J, Thorne PK, Martin JS, et al. Transcriptomic effects of metformin in skeletal muscle arteries of obese insulinresistant rats. Exp Biol Med (Maywood). 2017;242(6):617-624 doi: https://doi.org/10.1177/1535370216689825

71. Weijers RNM, Bekedam DJ. The Metformin Paradox. Curr Diabetes Rev. 2020;16(2):143-147. doi: https://doi.org/10.2174/1573399814666181 119145750

72. Schernthaner $\mathrm{G}$, Schernthaner $\mathrm{GH}$. The right place for metformin today. Diabetes Res Clin Pract. 2020;159:107946. doi: https://doi.org/10.1016/j.diabres.2019.107946

73. Schweighofer N, Genser B, Maerz W, et al. Intronic Variants in OCT1 are Associated with All-Cause and Cardiovascular Mortality in Metformin Users with Type 2 Diabetes. Diabetes Metab Syndr Obes. 2020;13:2069-2080. doi: https://doi.org/10.2147/DMSO.S235663

74. Unosson J, Wågsäter D, Bjarnegård N, et al. Metformin Prescription Associated with Reduced Abdominal Aortic Aneurysm Growth Rate and Reduced Chemokine Expression in a Swedish Cohort. Ann Vasc Surg. 2021;70:425-433. doi: https://doi.org/10.1016/j.avsg.2020.06.039

75. Zhang K, Yang W, Dai H, Deng Z. Cardiovascular risk following metformin treatment in patients with type 2 diabetes mellitus: Results from meta-analysis. Diabetes Res Clin Pract. 2020;160:108001. doi: https://doi.org/10.1016/j.diabres.2020.108001

76. Shoemaker AH, Chung ST, Fleischman A; Pediatric Endocrine Society Obesity Special Interest Group. Trends in pediatric obesity management, a survey from the Pediatric Endocrine Society Obesity Committee. J Pediatr Endocrinol Metab. 2020;33(4):469-472. doi: https://doi.org/10.1515/jpem-2019-0546.
77. Шестакова М.В., Викулова О.К., Исаков М.А.,Дедов И.И. Сахарный диабет и COVID-19: анализ клинических исходов по данным регистра сахарного диабета Российской Федерации // Проблемы эндокринологии. - 2020. - Т. 66. №1. - C. 35-46. [Shestakova MV, Vikulova OK, Isakov MA, Dedov II. Diabetes and COVID-19: analysis of the clinical outcomes according to the data of the russian diabetes registry. Problems of Endocrinology. 2020;66(1):35-46. (In Russ.)]. doi: https://doi.org/10.14341/probl12458

78. Шестакова М.В., Мокрышева Н.Г., Дедов И.И. Сахарный диабет в условиях вирусной пандемии COVID-19: особенности течения и лечения // Сахарный диабет. - 2020. - Т. 23. №2. - C. 132-139. [Shestakova MV, Mokrysheva NG, Dedov II. Course and treatment of diabetes mellitus in the context of COVID-19. Diabetes Mellitus. 2020;23(2):132-139. (In Russ.)]. doi: https://doi.org/10.14341/DM12418

79. Gao Y, Liu T, Zhong W, et al. Risk of Metformin in Patients With Type 2 Diabetes With COVID-19: A Preliminary Retrospective Report. Clin Trans/ Sci. 2020;13(6):1055-1059. doi: https://doi.org/10.1111/cts.12897

80. García-Calzón S, Perfilyev A, Martinell M, et al. Epigenetic markers associated with metformin response and intolerance in drugnaïve patients with type 2 diabetes. Sci Trans/ Med. 2020;12(561). doi: https://doi.org/10.1126/scitrans/med.aaz1803

81. Miyan Z, Waris N; MIBD. Association of vitamin $B_{12}$ deficiency in people with type 2 diabetes on metformin and without metformin: a multicenter study, Karachi, Pakistan. BMJ Open Diabetes Res Care. 2020;8(1):e001151. doi: https://doi.org/10.1136/bmjdrc-2019-001151

82. Wakeman M, Archer DT. Metformin and Micronutrient Status in Type 2 Diabetes: Does Polypharmacy Involving Acid-Suppressing Medications Affect Vitamin B12 Levels? Diabetes Metab Syndr Obes. 2020;13:2093-2108. doi: https://doi.org/10.2147/DMSO.S237454

83. Logie L, Harthill J, Patel K, et al. Cellular responses to the metalbinding properties of metformin. Diabetes. 2012;61(6):1423-1433. doi: https://doi.org/10.2337/db11-0961

84. Chen Y, Wu Y, Yang Y, et al. Transcriptomic and proteomic analysis of potential therapeutic target genes in the liver of metformin treated Sprague Dawley rats with type 2 diabetes mellitus. Int J Mol Med. 2018;41(6):3327-3341. doi: https://doi.org/10.3892/ijmm.2018.3535

85. Kaur B, Henry J. Micronutrient status in type 2 diabetes: a review. Adv Food Nutr Res. 2014;71:55-100. doi: https://doi.org/10.1016/B978-0-12-800270-4.00002-X

86. Siddiqui K, Bawazeer N, Scaria Joy S. Variation in Macro and Trace Elements in Progression of Type 2 Diabetes. Sci World J. 2014;2014:1-9. doi: https://doi.org/10.1155/2014/461591

87. Krysiak R, Kowalcze K, Okopień B. The impact of combination therapy with metformin and exogenous vitamin D on hypothalamic-pituitary-thyroid axis activity in women with autoimmune thyroiditis and high-normal thyrotropin levels. J Clin Pharm Ther. 2020;45(6):1382-1389. doi: https://doi.org/10.1111/jcpt.13233

88. Kanti G, Anadol-Schmitz E, Bobrov P, et al. Vitamin B12 and Folate Concentrations in Recent-onset Type 2 Diabetes and the Effect of Metformin Treatment. J Clin Endocrinol Metab. 2020;105(6):2222-2231. doi: https://doi.org/10.1210/clinem/dgaa150

89. Morgante G, Massaro MG, Scolaro V, et al. Metformin doses and body mass index: clinical outcomes in insulin resistant polycystic ovary syndrome women. Eur Rev Med Pharmacol Sci. 2020;24(15):8136-8142. doi: https://doi.org/10.26355/eurrev_202008_22500

90. Wu H, Esteve E, Tremaroli V, et al. Metformin alters the gut microbiome of individuals with treatment-naive type 2 diabetes, contributing to the therapeutic effects of the drug. Nat Med. 2017;23(7):850-858. doi: https://doi.org/10.1038/nm.4345

91. Nakajima H, Takewaki F, Hashimoto $Y$, et al. The Effects of Metformin on the Gut Microbiota of Patients with Type 2 Diabetes: A TwoCenter, Quasi-Experimental Study. Life (Basel). 2020;10(9):195. doi: https://doi.org/10.3390/life10090195

92. Vallianou NG, Stratigou T, Tsagarakis S. Metformin and gut microbiota: their interactions and their impact on diabetes. Hormones (Athens). 2019:18(2):141-144. doi: https://doi.org/10.1007/s42000-019-00093-w

93. Koh A, Manneras-Holm L, Yunn NO, et al. Microbial Imidazole Propionate Affects Responses to Metformin through p38yDependent Inhibitory AMPK Phosphorylation. Cell Metab. 2020;32(4):643-653. doi: https://doi.org/10.1016/j.cmet.2020.07.012 


\section{ИНФОРМАЦИЯ ОБ АВТОРАХ [AUTHORS INFO]}

* Кучер Аксана Николаевна, д.б.н., профессор [Aksana N. Kucher, PhD in Biology, Professor]; адрес: Pоссия, 634050, Томск, Набережная р. Ушайки, д. 10 [address: 10 Ushaika river embankment, 634050 Tomsk, Russia]; ORCID: http://orcid.org/0000-0003-3824-3641; Researcher ID: A-7789-2014; Scopus Author ID: 7004507293; eLibrary SPIN: 5251-2055; e-mail: aksana.kucher@medgenetics.ru

Бабушкина Надежда Петровна, к.б.н. [Nadezhda P. Babushkina, PhD in Biology]; ORCID: https://orcid.org/0000-0001-6133-8986; Researcher ID: B-3106-2014; Scopus Author ID: 39961071300; eLibrary SPIN: 4639-1490; e-mail: nad.babushkina@medgenetics.ru

\section{ЦИТИРОВАТЬ:}

Кучер А.Н., Бабушкина Н.П. Роль генетических и средовых факторов в детерминации ответа на метформин // Сахарный диабет. — 2021. - Т. 24. — №6. — C. 571-582. doi: https://doi.org/10.14341/DM12744

\section{TO CITE THIS ARTICLE:}

Kucher AN, Babushkina NP. Role of Genetic and Environmental Factors in Determining the Response to Metformin. Diabetes Mellitus. 2021;24(6):571-582. doi: https://doi.org/10.14341/DM12744 